\title{
A Comparative Seroprevalence Study of Toxocariasis in Hypereosinophilic and Apparently Healthy Individuals
}

\author{
Bahador Sarkari $^{1, *} ;$ Malihe Lari $^{2}$; Reza Shafiei $^{2}$; Seyed Mahmoud Sadjjadi ${ }^{2}$ \\ ${ }^{1}$ Basic Sciences in Infectious Diseases Research Center, Shiraz University of Medical Sciences, Shiraz, IR Iran \\ ${ }^{2}$ Department of Parasitology and Mycology, School of Medicine, Shiraz University of Medical Sciences, Shiraz, IR Iran \\ ${ }^{*}$ Corresponding author: Bahador Sarkari, Basic Sciences in Infectious Diseases Research Center, Shiraz University of Medical Sciences, Shiraz, IR Iran. Tel/Fax: +98-7112305291, \\ E-mail: sarkarib@sums.ac.ir
}

Received: January 28, 2014; Revised: February 28, 2014; Accepted: May 10, 2014

\begin{abstract}
Background: Parasitic invasion of tissues can elicit hypereosinophilia. Among helminthic infections, moderate to severe eosinophilia can be seen in toxocariasis as a pathophysiological response to a worm infection.

Objectives: The current study aimed to compare the rate of toxocariasis in normal and hypereosinophilic individuals in Shiraz, southern Iran.

Patients and Methods: Serum samples of 100 patients with hypereosinophilia (>10\%) and 100 normal individuals (without eosinophilia) were collected. Demographic information of the participants was recorded in a predesigned questionnaire during sample collection. Anti-Toxocara antibodies in the subjects' serum were evaluated by an ELISA.

Results: In the hypereosinophilic patients, 54 (54\%) were male, and 46 (46\%) were female, while in the normal individuals, 58 cases (58\%) were male, and $42(42 \%)$ were female. The differences in gender and age of the participants were insignificant $(\mathrm{P}=0.46$ and 0.59 respectively). Range of eosinophilia found in the patients was between 10-77\%. Anti-Toxocara antibodies were detected in the serum of 2 (2\%) of the hypereosinophilic patients and 3 (3\%) of the normal individuals. No significant correlation was found between hypereosinophilia and the presence of anti-Toxocara antibodies in this study $(\mathrm{P}=0.65)$.

Conclusions: Hypereosinophilia may be due to a variety of reasons and toxocariasis is just one of these. Appropriate tests should be carried out on patients in order to rule out a Toxocara infection in hypereosinophilic individuals.
\end{abstract}

Keywords: Toxocara; Prevalence; Iran

\section{Background}

Toxocariasis is a zoonotic helminthic infection caused by the larval stages of Toxocara canis and Toxocara cati, which are common roundworms found in dogs and cats (1). The disease mainly affects children under five yearsof-age. Human infection occurs through ingestion of embryonated eggs from the soil, dirty hands, uncooked vegetables, and paratenic hosts (2). The most widely recognized source of human infection is ingestion of contaminated soil followed by paratenic hosts (2-7). The released larvae from the eggs or paratenic hosts enter the circulation which then invade and form granulomas in different tissues, including; liver, lungs, eyes, brain and other tissues $(8,9)$. The prevalence of toxocariasis due to soil contamination with Toxocara spp. eggs is from $6.6 \%$ to $87.1 \%(10-14)$.

Inflammatory responses and eosinophilia are the most common signs of a Toxocara infection $(4,8)$. In helminthic diseases, moderate to severe eosinophilia occurs as a pathophysiologic response to the parasitic infection and toxocariasis is one of the main causes of eosinophilia, regardless of the presence of signs and symptoms or organ injury, in infected individuals (15). However, in many cases a peripheral eosinophilia is not connected to toxocariasis
(16). High seroprevalence rates of toxocariasis have been reported in hypereosinophilic patients. Seo and Yoon in a seroepidemiological study in Korea, reported that out of a total of 101 eosinophilic patients, 50.5\% were found to be positive by a Toxocara ELISA (17). In another study in Korea, the seroprevalence of toxocariasis among healthy people with eosinophilia was reported to be $67.0 \%$ (18). In a relatively similar study, the seroprevalence of toxocariasis among asymptomatic children with eosinophilia in Croatia was found to be 31\% (19). Maraghi et al. in a study conducted in Khuzestan, southern Iran, found that 19\% of eosinophilic individuals were seropositive for toxocariasis (20).

\section{Objectives}

The aim of the present study was to determinate and compare the rate of toxocariasis in normal and hypereosinophilic individuals in Shiraz, southern Iran.

\section{Patients and Methods}

In this cross-sectional study which was carried out during February 2010 to April 2012, serum samples were collected from 100 patients with hypereosinophilia and 100

Copyright (C) 2015, Pediartric Infections Research Center. This is an open-access article distributed under the terms of the Creative Commons Attribution-NonCommercial 4.0 International License (http://creativecommons.org/licenses/by-nc/4.0/) which permits copy and redistribute the material just in noncommercial usages, provided the original work is properly cited.. 
subjects with a normal eosinophil count (1-6\%), who were referred to the three main university affiliated hospitals in Shiraz, Fars Province, southern Iran. The control group was composed of outpatients who attended the outpatient clinics or hospitals for a routine checkup, or those who had no obvious infectious diseases.

Sample size was calculated based on the prevalence rate of a previously published study on the seroprevalence of toxocariasis in the region, and the sample size of similar studies. There were 21 patients with a peripheral blood eosinophilia of $10 \%$ or higher which were considered as hypereosinophilia (15). Demographic features of the participants were recorded in a predesigned questionnaire during sample collecting. Written informed consent was obtained from the participants, or their parent or guardian, where the participants were children. All sera were tested for IgG antibodies to Toxocara spp. by an enzyme-linked immunosorbent assay (ELISA, IBL/Germany) according to the manufacturer's instructions. The test utilized an immunoassay to detect antiToxocara canis excretory-secretory antigens (TES). Cutoff value was $10 \mathrm{U}$, while 9-11 U was considered as a gray zone, $<9$ U was negative, and $>11$ was positive. Based on the data sheet of the ELISA kit, the sensitivity and specificity of the kit are reported to be $>95 \%$. Collected data were analyzed by SPSS (Version 16.0. Chicago, SPSS Inc). Chi-square was used to compare the seroprevalence rate in the two groups.

\section{Results}

In the hypereosinophilic patients, 54 (54\%) were male, and 46 (46\%) were female, while in the normal individuals, 58 cases (58\%) were male, and 42 (42\%) were female. The differences in gender and age of the participants were insignificant ( $\mathrm{P}=0.46$ and 0.59 respectively). The range of eosinophilia found in the patients was between 10-77\%. Anti-Toxocara antibodies were detected in the serum of 2 (2\%) of the hypereosinophilic patients and 3 (3\%) of the normal individuals. No significant correlation was found between hypereosinophilia and the presence of anti-Toxocara antibodies in this study $(\mathrm{P}=0.65)$. More- over, no association was found between seropositivity to Toxocara and the age or sex of the participants $(\mathrm{P}=0.53$ and 0.49 , respectively). Table 1 shows the age distribution of the participants and seropositivity to toxocariasis.

\section{Discussion}

Human toxocariasis is a helminthic zoonotic disease with a worldwide distribution. Seroprevalence studies in different areas of the world have documented the rate of infection to be as high as $80 \%$ in some areas (1). In Iran, the seroprevalence of toxocariasis varies between $2.7 \%$ and 29.3\% in different areas of the country (21-24). Manifestations of toxocariasis are diverse and non-characteristic, and in many cases they are also asymptomatic. Like other tissue dwelling parasites, Toxocara induces moderate to severe eosinophilia in infected individuals $(1,8)$. Therefore, hypereosinophilia is a key finding for the diagnosis of toxocariasis when the other reasons for this symptom have been ruled out. Peripheral blood eosinophilia is one of the most important features of human toxocariasis. This phenomenon can be seen in visceral larva migrants (VLM). However, due to the low larval burden, eosinophilia is not present in ocular larva migrants (OLM) patients. Moreover, some toxocariasis patients may have toxocariasis without eosinophilia.

Hypergammaglobulinemia and elevated concentrations of total serum IgE are the other laboratory findings of toxocariasis. Patients with an increase in the concentration of serum total IgE $>500 \mathrm{IU} / \mathrm{mL}$, along with one or more clinical signs, might be considered as toxocariasis (25). In many studies conducted on the seroprevalence of toxocariasis, a higher rate of infection has been reported in eosinophilic patients in comparison with non-eosinophilic subjects. However, such correlation has not been seen in several other studies (17). In a study conducted by Maraghi et al. in the south of Iran, serum samples from 100 individuals with peripheral blood eosinophilia, and from another 100 individuals without eosinophilia, were evaluated for anti-Toxocara antibodies. Antibodies were found in 19 (19\%) of the eosinophilic individuals and only $1 \%$ of the control group (20). In another study, in Turkey,

\begin{tabular}{lcccc}
\hline \multicolumn{2}{l}{ Table 1. Age Distribution of the Study's Participants and Seropositivity to Toxocariasis } \\
\hline
\end{tabular}


IgG antibodies specific to Toxocara were detected in 114 (32.6\%) of the eosinophilic patients in comparison with $71(20.3 \%)$ of the non-eosinophilic individuals (26). In our study no difference was found between the seropositivity rates of toxocariasis between the two groups.

Detection of anti-Toxocara antibodies in a non-eosinophilic subject might be due to a past infection. Such findings have been reported in different studies where healthy subjects are seropositive for toxocariasis $(27,28)$. On the other hand, the low prevalence rate of toxocariasis in both eosinophilic and normal individuals in our study might be due to the age of the subjects, who were mainly over 50 years-of-age. In a previous study which was conducted in the region about 12 years ago, the prevalence of toxocariasis in children was $25.6 \%(24)$.

Our findings are consistent with a study by Kim et al. in South Korea, where they did not find a significant correlation between the IgG ELISA titer for Toxocara and the level of eosinophilia (17). In our study, no association was found between the sex or age of participants and seropositivity to toxocariasis. A comparable finding was reported by Dogan et al. in Turkey, where they reported a similar seropositivity rate of Toxocara antibodies between age groups and genders (29).

Further studies with a relatively large sample of patients, with and without eosinophilia, and composed of different age groups, are needed to clarify any association between hypereosinophilia and toxocariasis in the region. Hypereosinophilia might be due to increased infections with Toxocara larva and a low rate of infection might result in lower eosinophilia (30). Hypereosinophilia can occur for a variety of reasons and toxocariasis is only one of these, but testing should be carried out for patients with suspected toxocariasis to rule out a Toxocara infection in hypereosinophilic individuals.

\section{Acknowledgements}

We are thankful to the Office of Vice-Chancellor for Research of Shiraz University of Medical Sciences for their financial support.

\section{Authors' Contributions}

BS and SMS participated in the study design. BS contributed in statistical analysis of the data and drafted the manuscript. RSH and MR collected the samples and conducted the experiments. All authors read and approved the final manuscript.

\section{Funding/Support}

This study was financially supported by the Office of Vice-Chancellor for Research of Shiraz University of Medical Sciences (Grant No. 89-01-43-2701). The study has been the subject of a medical student thesis (Malihe Lari).

\section{References}

1. Rubinsky-Elefant G, Hirata CE, Yamamoto JH, Ferreira MU. Human toxocariasis: diagnosis, worldwide seroprevalences and clinical expression of the systemic and ocular forms. Ann Trop Med Parasitol. 2010;104(1):3-23.

2. Azizi S, Oryan A, Sadjjadi SM, Zibaei M. Histopathologic changes and larval recovery of Toxocara cati in experimentally infected chickens. Parasitol Res. 2007;102(1):47-52.

3. Fisher M. Toxocara cati: an underestimated zoonotic agent. Trends Parasitol. 2003;19(4):167-70.

4. Toxocara: the enigmatic parasite.Wallingford UK: CABI International; 2006. P. 58-73.

5. Roddie G, Stafford P, Holland C, Wolfe A. Contamination of dog hair with eggs of Toxocara canis. Vet Parasitol. 2008;152(12):85-93.

6. Taira K, Saitoh Y, Kapel CM. Toxocara cati larvae persist and retain high infectivity in muscles of experimentally infected chickens. Vet Parasitol. 2011;180(3-4):287-91.

7. Noh Y, Hong ST, Yun JY, Park HK, Oh JH, Kim YE, et al. Meningitis by Toxocara canis after ingestion of raw ostrich liver. J Korean Med Sci. 2012;27(9):1105-8.

8. Despommier D. Toxocariasis: clinical aspects, epidemiology, medical ecology, and molecular aspects. Clin Microbiol Rev. 2003;16(2):265-72.

9. Zibaei M, Sadjjadi SM, Uga S. Experimental Toxocara cati infection in gerbils and rats. Korean J Parasitol. 2010;48(4):331-3.

10. Chorazy ML, Richardson DJ. A survey of environmental contamination with ascarid ova, Wallingford, Connecticut. Vector Borne Zoonotic Dis. 2005;5(1):33-9.

11. Guimaraes AM, Alves EG, de Rezende GF, Rodrigues MC. [Toxocara sp. eggs and Ancylostoma sp. larva in public parks, Brazil]. Rev Saude Publica. 2005;39(2):293-5.

12. Rokicki J, Kucharska AP, Dzido J, Karczewska D. [Contamination of playgrounds in Gdansk city with parasite eggs]. Wiad Parazytol. 2007;53(3):227-30.

13. Tiyo R, Guedes TA, Falavigna DL, Falavigna-Guilherme AL. Seasonal contamination of public squares and lawns by parasites with zoonotic potential in southern Brazil. J Helminthol. 2008;82(1):1-6.

14. Zibaei M, Uga S. Contamination by Toxocara spp. Eggs in sandpits in Kobe Japan. J Environ Cont Tech. 2008;26:32-7.

15. Kwon NH, Oh MJ, Lee SP, Lee BJ, Choi DC. The prevalence and diagnostic value of toxocariasis in unknown eosinophilia. Ann $\mathrm{He}$ matol. 2006;85(4):233-8.

16. Magnaval JF, Glickman LT, Dorchies P, Morassin B. Highlights of human toxocariasis. Korean J Parasitol. 2001;39(1):1-11.

17. Seo M, Yoon SC. A seroepidemiological survey of toxocariasis among eosinophilia patients in Chungcheongnam-do. Korean J Parasitol. 2012;50(3):249-51

18. Kim YH, Huh S, Chung YB. Seroprevalence of toxocariasis among healthy people with eosinophilia. Korean J Parasitol. 2008;46(1):29-32.

19. Sviben M, Cavlek TV, Missoni EM, Galinovic GM. Seroprevalence of Toxocara canis infection among asymptomatic children with eosinophilia in Croatia. JHelminthol. 2009;83(4):369-71

20. Maraghi S, Rafiei A, Hajihossein R, Sadjjadi SM. Seroprevalence of toxocariasis in hypereosinophilic individuals in Ahwaz, southwestern Iran. J Helminthol. 2012;86(2):241-4.

21. Nourian AA, Amiri M, Ataeian A, Haniloo A, Mosavinasab SN, Badali H. Seroepidemiological study for toxocariasis among children in Zanjan-northwest of Iran. PakJBiol Sci. 2008;11(14):1844-7.

22. Akhlaghi L, Ourmazdi H, Sarafnia A, Vaziri S, Jadidian K, Leghaii Z [An Investigation on the Toxocariasis Seroprevalence in Children (2-12 Years Old) from Mahidasht Area of Kermanshah Province (2003-2004)]. Razi JMed Sci. 2006;13(52):41-8.

23. Fallah MAA, Taherkhani H. Seroprevalence of toxocariasis in children aged 1-9 years in Hamadan - 2002.J Zanjan Univ Med Sci Health Serv. 2005

24. Alavi SM, Rahdar M, Salmanzadeh S, Nikkhuy AR. Determination of seroprevalence rate of Toxocara canis in 6-15 years aged rural and urban school children in Ahvaz, Iran. Jundishapur Sci Med J. 2011;10:239-48. 
25. Watthanakulpanich D. Diagnostic trends of human toxocariasis. J Trop Med Parasitol. 2010;33:44-52.

26. Karadam SY, Ertug S, Ertabaklar H, Okyay P. The comparison of IgG antibodies specific to Toxocara spp. among eosinophilic and non-eosinophilic groups. New Microbiol. 2008;31(1):113-6.

27. Choi D, Lim JH, Choi DC, Lee KS, Paik SW, Kim SH, et al. Transmission of Toxocara canis via ingestion of raw cow liver: a cross-sectional study in healthy adults. Korean J Parasitol. 2012;50(1):23-7.

28. Park HY, Lee SU, Huh S, Kong Y, Magnaval JF. A seroepidemiological survey for toxocariasis in apparently healthy residents in
Gangwon-do, Korea. Korean JParasitol. 2002;40(3):113-7.

29. Sadjjadi SM, Khosravi M, Mehrabani D, Orya A. Seroprevalence of Toxocara infection in school children in Shiraz, southern Iran. Trop Pediatr. 2000;46::327-30.

30. Dogan N, Dinleyici EC, Bor O, Toz SO, Ozbel Y. Seroepidemiological survey for Toxocara canis infection in the northwestern part of Turkey. Turkiye Parazitol Derg. 2007;31(4):288-91.

31. Overgaauw PA, van Knapen F. Veterinary and public health aspects of Toxocara spp. Vet Parasitol. 2013;193(4):398-403. 